\title{
A NOTE ON L-FUZZY BAGS AND THEIR EXPECTED VALUES
}

\author{
Fateme Kouchakinejad - MashaAllah Mashinchi — Radko Mesiar
}

\begin{abstract}
A definition of L-fuzzy bags is introduced and studied. In this approach, according to the concept given by M. Delgado et al. (2009), each bag has two parts: function and summary information. Then, the definition of L-fuzzy bag expected value is introduced. In the case $L=[0,1]$, several integral-based fuzzy bag expected values are prepared. By some examples, the new concepts are illustrated.
\end{abstract}

\section{Introduction}

The initial notion of bags, an alternative name for the multisets, was introduced by Yager [9] as an algebraic set-like structure where an element can appear more than once. So far, several works have been done using this new concept. Moreover, bags have been employed in practice, for instance: in flexible querying, representation of relational information, decision problem analysis, criminal career analysis, and even in such a field as biology.

However, due to some existing drawbacks in the first definition of bags [9], the necessity of a revision of this notion revealed. The proposed definition by D elg a d o et al. [2] has corrected these drawbacks. By some examples, they showed that the given definition of bags by $\mathrm{Y}$ ag e $\mathrm{r}$ has some deficiencies and it was not well suited for representing and reasoning with real-world information. Then, they proposed new definitions of bags and fuzzy bags.

As it is shown in [5], the lattice of all fuzzy bags defined by D elgado et al. 2] is a complete Boolean algebra which is not compatible with the nature of fuzziness. Improving this incompatibility, in the present paper, we quote a revised definition of fuzzy bags as a special case of L-fuzzy bags based on the proposed definition of bags in [2]. Also we introduce the concept of the L-fuzzy bag expected value.

(C) 2016 Mathematical Institute, Slovak Academy of Sciences. 2010 Mathematics Subject Classification: 03B52, 94 D05.

Keywords: bags, expected value, L-fuzzy bags.

Research of the third author was supported by the grant VEGA 1/0420/15. 


\section{Preliminaries}

In this section, some basic concepts which are needed in the sequel are given. For more details, see [2].

Definition 1 ([2]). Let $P$ and $O$ be two universes (sets) called "properties" and "objects", respectively. A (crisp) bag $\mathcal{B}^{f}$ is a pair $\left(f, B^{f}\right)$, where $f: P \rightarrow \mathcal{P}(O)$ is a function and $B^{f}$ is the following subset of $P \times \mathcal{N}_{0}$

$$
B^{f}=\{(p, \operatorname{card}(f(p))) \mid p \in P\} .
$$

Here, $\mathcal{N}_{0}=\{0,1, \ldots\}=\{0\} \cup \mathcal{N}$, where $\mathcal{N}$ is the set of natural numbers, $\mathcal{P}(O)$ is the power set of $O, \operatorname{card}(X)$ is the cardinality of set $X$.

We will use the convention here that $\operatorname{card}(\emptyset)=0$.

In this characterization, a bag $\mathcal{B}^{f}$ consists of two parts. The first one is the function $f$ that can be seen as an information source about the relation between objects and properties. The second part $B^{f}$ is a summary of the information in $f$ obtained by means of the count operation $\operatorname{card}($.$) . This summary$ corresponds to the classical view of bags in the sense of [9].

Notation 1. We set $\mathbf{B}(P, O)$ as the set of all bags $\mathcal{B}^{f}=\left(f, B^{f}\right)$ defined in Definition 1 .

Definition 2. Define $\mathcal{B}^{0}=\left(0, B^{0}\right)$ and $\mathcal{B}^{1}=\left(1, B^{1}\right)$, where $0(p)=\emptyset, 1(p)=O$ for all $p \in P, B^{0}=\{(p, 0), p \in P\}$ and $B^{1}=\{(p, \operatorname{card}(O)), p \in P\}$. Clearly, $\mathcal{B}^{0}, \mathcal{B}^{1} \in \mathbf{B}(P, O)$.

Example 1 ([2]). Let $O=\{$ John, Ana, Bill, Tom, Sue, Stan, Ben $\}$ and $P=\{17,21,27,35\}$ be the set of objects and the set of properties, respectively. Let $f: P \rightarrow \mathcal{P}(O)$ be the function in Table 1 with $f(p) \subseteq O$ for all $p \in P$.

TABle 1. Function: age-people.

\begin{tabular}{|c|cccc|}
\hline$p$ & 17 & 21 & 27 & 35 \\
\hline$f(p)$ & $\{$ Bill, Sue $\}$ & $\{$ John, Tom, Stan $\}$ & $\emptyset$ & $\{$ Ben $\}$ \\
\hline
\end{tabular}

So, we can define bag $\mathcal{B}^{f}=\left(f, B^{f}\right)$, where $B^{f}=\{(17,2),(21,3),(27,0),(35,1)\}$.

Definition 3 ([2]). a) A bag $\mathcal{B}^{f}$ is a sub bag of $\mathcal{B}^{g}$, denoted by $\mathcal{B}^{f} \sqsubseteq \mathcal{B}^{g}$ if $f(p) \subseteq g(p)$ for all $p \in P$.

b) Two bags $\mathcal{B}^{f}$ and $\mathcal{B}^{g}$ are equal, denoted by $\mathcal{B}^{f}=\mathcal{B}^{g}$ if $\mathcal{B}^{f} \sqsubseteq \mathcal{B}^{g}$ and $\mathcal{B}^{g} \sqsubseteq \mathcal{B}^{f}$.

In the next section, we review the concept of fuzzy bags and quote some results about them. For more details see [7] and [8]. 


\section{A NOTE ON L-FUZZY BAGS AND THEIR EXPECTED VALUES}

\section{L-fuzzy bags}

In what follows, $O$ is the set of all objects, $L$ is a complete lattice and $\mathcal{F}_{L}(O)=$ $\{A \mid A: O \rightarrow L\}$ is the set of all L-fuzzy subsets of $O$. In the case of $L=[0,1]$, we write $\mathcal{F}(O)$. Also $i \in I_{n}=\{1,2, \ldots, n\}$, where $n \in \mathcal{N}$.

Definition $4([8])$. An L-fuzzy bag $\tilde{\mathcal{B}}^{\tilde{f}}$ is a pair $\left(\tilde{f}, B^{\tilde{f}}\right)$, where $\tilde{f}: P \rightarrow \mathcal{F}_{L}(O)$ is a function and $B^{f}$ is the following subset of $P \times L \times \mathcal{N}_{0}$

where

$$
B^{\tilde{f}}=\left\{\left(p, \delta, \operatorname{card}\left(O_{\delta}^{p}\right)\right) \mid p \in P, \delta \in L\right\},
$$

$$
O_{\delta}^{p}=\{o \in O \mid \tilde{f}(p)(o)=\delta\} .
$$

Obviously, a bag is a particular case of the L-fuzzy bag, where for all $p \in P$, $\tilde{f}(p)$ is a crisp subset of $O$. Similar to bags, the L-fuzzy bag $\tilde{\mathcal{B}}^{\tilde{f}}$ consists of two parts. The first one is the function $\tilde{f}$ that can be seen as an information source about the relation between objects and properties. The second part $B^{\tilde{f}}$ is a summary of the information in $\tilde{f}$ obtained by means of the count operation $\operatorname{card}($.$) .$

Note $1([8])$. In the case that $L=[0,1]$, the defined L-fuzzy bag in Definition 4 is called fuzzy bag.

Here, the concept of L-fuzzy bag is illustrated by an example.

Example $2(8])$. Let $L=[0,1], O=\{$ Ben, Sue, Tom, John, Stan, Bill, Kim, Ana, Sara $\}$ and $P=\{$ young, middle age, old $\}$ is the set of some linguistic descriptions of age. Let the degrees of membership of all $o \in O$ in the set of each property $p \in P$ be given as in Table 2.

TABLE 2. The degrees of memberships for Example 2

\begin{tabular}{|c|c|c|c|c|c|c|c|c|c|}
\hline $\mathrm{p}$ & Ben & Sue & Tom & John & Stan & Bill & Kim & Ana & Sara \\
\hline young & 0.7 & 0.2 & 0.4 & 0.0 & 0.7 & 0.4 & 0.2 & 0.7 & 0.1 \\
middle age & 0.3 & 0.8 & 0.7 & 0.3 & 0.3 & 0.7 & 0.8 & 0.3 & 0.5 \\
old & 0.1 & 0.2 & 0.1 & 0.9 & 0.1 & 0.1 & 0.2 & 0.1 & 0.5 \\
\hline
\end{tabular}

So, by Definition 4 , we can define fuzzy bag $\tilde{\mathcal{B}}^{\tilde{f}}=\left(\tilde{f}, B^{\tilde{f}}\right)$, where

$$
\begin{aligned}
\tilde{f}(\text { young }) & =\left\{\frac{0.7}{\text { Ben }}, \frac{0.2}{\text { Sue }}, \frac{0.4}{\text { Tom }}, \frac{0.7}{\text { Stan }}, \frac{0.4}{\text { Bill }}, \frac{0.2}{\text { Kim }}, \frac{0.7}{\text { Ana }}, \frac{0.1}{\text { Sara }}\right\}, \\
\tilde{f}(\text { middle age }) & =\left\{\frac{0.3}{\text { Ben }}, \frac{0.8}{\text { Sue }}, \frac{0.7}{\text { Tom }}, \frac{0.3}{\text { John }}, \frac{0.3}{\text { Stan }}, \frac{0.7}{\text { Bill }}, \frac{0.8}{\text { Kim }}, \frac{0.3}{\text { Ana }}, \frac{0.5}{\text { Sara }}\right\}, \\
\tilde{f}(\text { old }) & =\left\{\frac{0.1}{\text { Ben }}, \frac{0.2}{\text { Sue }}, \frac{0.1}{\text { Tom }}, \frac{0.9}{\text { John }}, \frac{0.1}{\text { Stan }}, \frac{0.1}{\text { Bill }}, \frac{0.2}{\text { Kim }}, \frac{0.1}{\text { Ana }}, \frac{0.5}{\text { Sara }}\right\},
\end{aligned}
$$


and

$B^{\tilde{f}}=\{$ (young, 0.7, 3), (young, 0.4, 2), (young, 0.2, 2), (young, 0.1, 1),

(middle age, $0.8,2$ ), (middle age, 0.7,2), (middle age, 0.5, 1), (middle age, $0.3,4$ ), (old, 0.9, 1), (old, 0.5, 1), (old, 0.2, 2), (old, 0.1, 5)\}.

Remark 1 ( 8$]$ ). As it can be seen, the more important part of an L-fuzzy bag is information function $\tilde{f}$. Therefore, it is possible to study the properties of L-fuzzy bags just by considering their information functions.

Notation $2([8])$. We set $\tilde{\mathbf{B}}_{L}(P, O)$ as the set of all L-fuzzy bags $\tilde{\mathcal{B}}^{\tilde{f}}=\left(\tilde{f}, B^{\tilde{f}}\right)$. Where, $\tilde{f}: P \rightarrow \mathcal{F}_{L}(O)$ and $B^{\tilde{f}}$ are as defined in Definition 4. Also we set $\tilde{\mathbf{B}}(P, O)$ as the set of all fuzzy bags. Clearly, $\mathbf{B}(P, O) \subseteq \tilde{\mathbf{B}}(P, O) \subseteq \tilde{\mathbf{B}}_{L}(P, O)$.

Here, we define intersection and union of L-fuzzy bags.

Definition 5. Let $\tilde{\mathcal{B}}^{\tilde{f}_{i}} \in \tilde{\mathbf{B}}_{L}\left(P_{i}, O_{i}\right)$ for all $i \in I_{n}$ be given L-fuzzy bags, $\bar{O}=\cap_{i \in I_{n}} O_{i}$ and $\bar{P}=\cap_{i \in I_{n}} P_{i}$. Then, their intersection is L-fuzzy bag

where

$$
\cap_{i \in I_{n}} \tilde{\mathcal{B}}^{\tilde{f}_{i}}=\left(\cap_{i \in I_{n}} \tilde{f}_{i}, B^{\cap_{i \in I_{n}} \tilde{f}_{i}}\right) \text {, }
$$

$$
\cap_{i \in I_{n}} \tilde{f}_{i}: \bar{P} \rightarrow \mathcal{F}_{L}(\bar{O}) \text { such that }\left(\cap_{i \in I_{n}} \tilde{f}_{i}\right)(p)=\bigcap_{i \in I_{n}} \tilde{f}_{i}(p)
$$

Also

where

$$
B^{\bigcap_{i \in I_{n}} \tilde{f}_{i}}=\left\{\left(p, \delta, \operatorname{card}\left(O_{\delta}^{p}\right)\right) \mid p \in \bar{P}, \delta \in L\right\}
$$

$$
O_{\delta}^{p}=\left\{o \in \bar{O} \mid\left(\cap_{i \in I_{n}} \tilde{f}_{i}\right)(p)(o)=\delta\right\}
$$

Note that by Definition $4, \cap_{i \in I_{n}} \tilde{\mathcal{B}}^{\tilde{f}_{i}}=\tilde{\mathcal{B}}^{\bigcap_{i \in I_{n}}} \tilde{f}_{i}$.

Definition 6. Let $\tilde{\mathcal{B}}^{\tilde{f}_{i}} \in \tilde{\mathbf{B}}_{L}\left(P_{i}, O_{i}\right)$ for all $i \in I_{n}$ be given L-fuzzy bags, $\bar{O}=\cup_{i \in I_{n}} O_{i}$ and $\bar{P}=\cup_{i \in I_{n}} P_{i}$. Then, their union is L-fuzzy bag

where

$$
\cup_{i \in I_{n}} \tilde{\mathcal{B}}^{\tilde{f}_{i}}=\left(\cup_{i \in I_{n}} \tilde{f}_{i}, B^{\cup_{i \in I_{n}} \tilde{f}_{i}}\right) \text {, }
$$

Also

$$
\cup_{i \in I_{n}} \tilde{f}_{i}: \bar{P} \rightarrow \mathcal{F}_{L}(\bar{O}) \text { such that }\left(\cup_{i \in I_{n}} \tilde{f}_{i}\right)(p)=\cup_{i \in I_{n}} \tilde{f}_{i}(p)
$$

where

$$
B^{\cup_{i \in I_{n}} \tilde{f}_{i}}=\left\{\left(p, \delta, \operatorname{card}\left(O_{\delta}^{p}\right)\right) \mid p \in \bar{P}, \delta \in L\right\},
$$

$$
O_{\delta}^{p}=\left\{o \in \bar{O} \mid\left(\cup_{i \in I_{n}} \tilde{f}_{i}\right)(p)(o)=\delta\right\}
$$

Note that by Definition 4, $\cup_{i \in I_{n}} \tilde{\mathcal{B}}^{\tilde{f}_{i}}=\tilde{\mathcal{B}}^{\cup_{i \in I_{n}}} \tilde{f}_{i}$. 


\section{A NOTE ON L-FUZZY BAGS AND THEIR EXPECTED VALUES}

The following definition equipes the set of all L-fuzzy bags with an order.

Definition 7 ([8]). An L-fuzzy bag $\tilde{\mathcal{B}}^{\tilde{f}}$ is an L-fuzzy sub bag of $\tilde{\mathcal{B}}^{\tilde{g}}$, denoted by $\tilde{\mathcal{B}} \tilde{\tilde{f}} \tilde{\subseteq} \tilde{\mathcal{B}}^{\tilde{g}}$ if and only if $\tilde{f}(p) \tilde{\subseteq} \tilde{g}(p)$ for all $p \in P$. That means $\tilde{\mathcal{B}} \tilde{f} \tilde{\subseteq} \tilde{\mathcal{B}} \tilde{g}$ if and only if for all $p \in P, \tilde{f}(p)$ be an L-fuzzy subset of $\tilde{g}(p)$.

Definition 8 ([8]). Two L-fuzzy bags $\tilde{\mathcal{B}}^{\tilde{f}}$ and $\tilde{\mathcal{B}}^{\tilde{g}}$ are equal, denoted by $\tilde{\mathcal{B}}^{\tilde{f}} \cong \tilde{\mathcal{B}}^{\tilde{g}}$ if $\tilde{\mathcal{B}}^{\tilde{f}} \tilde{\underline{\mathcal{B}}} \tilde{\mathcal{B}}^{\tilde{g}}$ and $\tilde{\mathcal{B}} \tilde{g} \tilde{\check{E}} \tilde{\mathcal{B}} \tilde{f}$ that means if $\tilde{f}=\tilde{g}$.

The next theorem gives some useful results about L-fuzzy bags.

Theorem 1 ([8]). Operations $\cup$ and $\cap$ in $\tilde{\boldsymbol{B}}_{L}(P, O)$ satisfy the laws of idempotency, commutativity, associativity and distributivity. Moreover, $\mathcal{B}^{0}$ is neutral for operation $\cup$ and $\mathcal{B}^{1}$ is neutral for operation $\cap$.

In the following definition, we introduce the concept of complement of an L-fuzzy bag.

Definition 9 ([8]). Let $\eta: L \rightarrow L$ be a fixed strong negation [1], this means an involutive decreasing bijection. Consider $\tilde{\mathcal{B}}^{\tilde{f}}=\left(\tilde{f}, B^{\tilde{f}}\right)$. Then, the $\eta$-complement of $\tilde{\mathcal{B}}^{\tilde{f}}$ is the L-fuzzy bag $\left(\tilde{\mathcal{B}}^{\tilde{f}}\right)^{c}=\left(\tilde{f}^{c}, B^{\tilde{f}^{c}}\right)$, where $\tilde{f}^{c}: P \rightarrow \mathcal{F}_{L}(O)$ such that $\tilde{f}^{c}(p)(o)=\eta(\tilde{f}(p)(o))$ for all $p \in P$ and $o \in O$.

Note that by Definition目 $\left(\tilde{\mathcal{B}}^{\tilde{f}}\right)^{c}=\tilde{\mathcal{B}}^{\tilde{f}^{c}}$.

Note 2 ( 8$]$ ). In Definition 9, if $L=[0,1]$ and $\eta$ is the standard negation, $\eta(x)=1-x$ for all $x \in[0,1][1]$, then $\tilde{\mathcal{B}}^{\tilde{f}^{c}}$ is called complement of $\tilde{\mathcal{B}}^{\tilde{f}}$.

Example 3 (7). Consider the fuzzy bag of Example2. The complement of this fuzzy bag is $\tilde{\mathcal{B}}^{c} \tilde{f}^{c}=\left(\tilde{f}^{c}, B^{\tilde{f}^{c}}\right)$, where

$$
\begin{aligned}
& \left.\tilde{f}^{c} \text { (young }\right)=\left\{\frac{0.3}{\text { Ben }}, \frac{0.8}{\text { Sue }}, \frac{0.6}{\text { Tom }}, \frac{1.0}{\text { John }}, \frac{0.3}{\text { Stan }}, \frac{0.6}{\text { Bill }}, \frac{0.8}{\text { Kim }}, \frac{0.3}{\text { Ana }}, \frac{0.9}{\text { Sara }}\right\}, \\
& \tilde{f}^{c} \text { (middle age) }=\left\{\frac{0.7}{\text { Ben }}, \frac{0.2}{\text { Sue }}, \frac{0.3}{\text { Tom }}, \frac{0.7}{\text { John }}, \frac{0.7}{\text { Stan }}, \frac{0.3}{\text { Bill }}, \frac{0.2}{\text { Kim }}, \frac{0.7}{\text { Ana }}, \frac{0.5}{\text { Sara }}\right\} \text {, } \\
& \tilde{f}^{c} \text { (old) }=\left\{\frac{0.9}{\text { Ben }}, \frac{0.8}{\text { Sue }}, \frac{0.9}{\text { Tom }}, \frac{0.1}{\text { John }}, \frac{0.9}{\text { Stan }}, \frac{0.9}{\text { Bill }}, \frac{0.8}{\text { Kim }}, \frac{0.9}{\text { Ana }}, \frac{0.5}{\text { Sara }}\right\} \text {, }
\end{aligned}
$$

and

$B^{\tilde{f}^{c}}=\{$ (young, 1.0, 1), (young, 0.9, 1), (young, 0.8, 2), (young, 0.6, 2), (young, 0.3, 3),

(middle age, $0.7,4$ ), (middle age, $0.5,1$ ), (middle age, $0.3,2$ ), (middle age, $0.2,2$ ), (old, $0.9,5),($ old $, 0.8,2)$, (old, $0.5,1)$, (old, $0.1,1)\}$. 


\section{Expected value of an L-fuzzy bag event}

In this section, we determine the "size" of a (L-fuzzy) bag via the definition of (L-fuzzy) bag expected value. To have some background of expected values, see [3]. Also please c.f. [6].

Definition 10. The bag expected value is the function $\mathrm{BEV}: \mathbf{B}(P, O) \rightarrow[0,1]$ with the following properties:

(i) $\operatorname{BEV}\left(\mathcal{B}^{0}\right)=0$ and $\operatorname{BEV}\left(\mathcal{B}^{1}\right)=1$;

(boundary conditions).

(ii) $\operatorname{BEV}\left(\mathcal{B}^{f}\right) \leq \operatorname{BEV}\left(\mathcal{B}^{g}\right)$ whenever $\mathcal{B}^{f} \sqsubseteq \mathcal{B}^{g} ; \quad$ (monotone non-decreasing).

Similar to the crisp case, we can have the fuzzy version.

Definition 11. An L-fuzzy bag expected value is the function LFBEV : $\tilde{\mathbf{B}}_{L}(P, O) \rightarrow[0,1]$ which satisfies the following conditions

(i) $\operatorname{LFBEV}\left(\mathcal{B}^{0}\right)=0$ and $\operatorname{LFBEV}\left(\mathcal{B}^{1}\right)=1 ; \quad$ (boundary conditions).

(ii) $\operatorname{LFBEV}\left(\tilde{\mathcal{B}}^{\tilde{f}}\right) \leq \operatorname{LFBEV}\left(\tilde{\mathcal{B}}^{\tilde{g}}\right)$ whenever $\tilde{\mathcal{B}}^{\tilde{f}} \tilde{\check{E}} \tilde{\mathcal{B}}^{\tilde{g}} ; \quad$ (monotone non-decreasing). In the case of $L=[0,1]$, we have fuzzy bag expected value and we write FBEV.

Remark 2. The restriction of an L-fuzzy bag expected value to the crisp bags is bag expected value, i.e., $\left.\mathrm{LFBEV}\right|_{\mathbf{B}(P, O)}=\mathrm{BEV}$.

Employing different measures, one can have various (L-fuzzy) bag expected values as it can be seen in the following examples.

Example 4. Considering probability measure $\operatorname{Pr}$ on $P \times O$, we can define the following fuzzy bag expected value

$$
\mathrm{FBEV}_{\operatorname{Pr}}\left(\tilde{\mathcal{B}}^{\tilde{f}}\right)=\sum_{\substack{p \in P \\ o \in O}} \operatorname{Pr}(\{(p, o)\}) \times \tilde{f}(p)(o) .
$$

Note that equation (3) is the Lebegue integral based expected value. One may compare it to expectation of a fuzzy set as defined in [10]. In the case of crisp bag, we have

$$
\mathrm{BEV}_{\operatorname{Pr}}\left(\mathcal{B}^{f}\right)=\sum_{\substack{p \in P \\ o \in f(p)}} \operatorname{Pr}(\{(p, o)\}) .
$$

Example 5. Considering possibility measure $\Pi$ on $P \times O$, we can define the following L-fuzzy bag expected value

$$
\operatorname{LFBEV}_{\Pi}\left(\tilde{\mathcal{B}}^{\tilde{f}}\right)=\bigvee_{\substack{p \in P \\ o \in O}} \Pi(\{(p, o)\}) \wedge \tilde{f}(p)(o)
$$




\section{A NOTE ON L-FUZZY BAGS AND THEIR EXPECTED VALUES}

Note that equation (5) is the Sugeno integral based expected value. In the case of crisp bag, we have

$$
\left.\operatorname{BEV}_{\Pi}\left(\mathcal{B}^{f}\right)=\bigvee_{\substack{p \in P \\ o \in f(p)}} \Pi((p, o)\}\right) .
$$

Remark 3. Given a monotone measure $\mu$ on $P \times O$, one can consider any universal integral $I$ acting on $[0,1]$, see [4], to define a fuzzy expectation $\operatorname{FBEV}_{I, \mu}\left(\tilde{\mathcal{B}}^{\tilde{f}}\right)=$ $I(\mu, \tilde{f})$. Then, (5) is based on the Sugeno integral, but we can consider the Choquet, the Shilkret and other integrals, too.

\section{Conclusion}

Employing the proposed definition of bags by Delgado et al., which is improved version of Yager's one, a new definition of L-fuzzy bags has been given. Then, the new concept of L-fuzzy bag expected value has been introduced. When considering $L=[0,1]$, several integral-based fuzzy bag expected values were introduced, too.

\section{REFERENCES}

[1] Beliakov, G.-PRADERA, A.-CAlVO, T.: Aggregation Functions: A Guide for Practitioners. Berlin, Springer, 2007.

[2] DELGADO, M.-RUIZ, M. D.-SANCHEZ, D.: RL-bags: a conceptual, level-based approach to fuzzy bags, Fuzzy Sets and Systems 208 (2012), 111-128.

[3] KLEMENT, E. P.-MESIAR, R.: On the expected value of fuzzy events, Internat. J. Uncertain. 23 (2015), 57-74.

[4] KLEMENT, E. P.-MESIAR, R.-PAP, E.: A universal integral as common frame for Choquet and Sugeno integral, IEEE Trans. Fuzzy Syst. 18 (2010), 178-187.

[5] KOUCHAKINEJAD, F.-MASHINCHI, M.: Algebraic structure of bags and fuzzy bags, in: The 46th Annual Iranian Mathematics Conference-AIMC '46, Yazd University, Yazd, Iran, 2015, pp. 1295-1298.

[6] KOUCHAKINEJAD, F.-MASHINCHI, M.-MESIAR, R.: Fuzzy bag expected values, in: The 13th Iranian Statistics Conference, Shahid Bahonar University of Kerman, Kerman, Iran, 2016 (accepted).

[7] KOUCHAKINEJAD, F.-MASHINCHI, M.-MESIAR, R.: Fuzzy bags, in: Uncertainty Modelling 2015, Union of Slovak Mathematicians and Physicists, Bratislava, 2016, 61-66.

[8] KOUCHAKINEJAD, F.-MASHINCHI, M.-MESIAR, R.: On algebraic structure of L-fuzzy bags, Int. J. Intell. Syst. 31 (2016), no. 11, 1035-1055.

[9] YAGER R. R.: On the theory of bags, Int. J. Gen. Syst. 13 (1986), 23-37. 


\section{FATEME KOUCHAKINEJAD - MASHAALLAH MASHINCHI — RADKO MESIAR}

[10] ZADEH L. A.: Probability measures of fuzzy events, J. Math. Anal. Appl. 23 (1968), 421-427.

Received July 15, 2016

Fateme Kouchakinejad

Department of Mathematics

Graduate University of Advanced Technology

Kerman

IRAN

E-mail: kouchakinezhad@gmail.com

Mashaallah Mashinchi

Department of Statistics

Faculty of Mathematics and

Computer Sciences

Shahid Bahonar University of Kerman

Kerman

IRAN

E-mail: mashinchi@uk.ac.ir

Radko Mesiar

Department of Mathematics and

Descriptive Geometry

Faculty of Civil Engineering

Slovak University of Technology in Bratislava

Radlinského 11

SK-810-05 Bratislava

SLOVAKIA

E-mail: radko.mesiar@stuba.sk 